\title{
COMPOSITIONAL CONTROL OF ANISOTROPY OF REMANENT AND INDUCED MAGNETIZATION IN SYNTHETIC SAMPLES \\ Mary Johns ${ }^{1}$ and Mike Jackson \\ Department of Geology and Geophysics, University of Minnesota
}

\begin{abstract}
Anisotropy of remanent and induced magnetization was found to be compositionally controlled in synthetic samples of varying proportions of chlorite (a platy paramagnet), manganese oxide (an isotropic paramagnet), and magnetite (an anisotropic ferrimagnet). Grains were magnetically aligned in a $100 \mathrm{mT}$ field, then uniaxially compressed at $27.6 \mathrm{MPa}$ to produce an L-S fabric. Anisotropy of anhysteretic susceptibility (AAS) and anisotropy of magnetic low-field susceptibility (AMS) are coaxial, and anhysteretic susceptibility is consistently more anisotropic than low-field susceptibility. AMS increases with the addition of oblate magnetite, but decreases with addition of isotropic $\mathrm{Mn}_{2} \mathrm{O}_{3}$ to chlorite. These experiments demonstrate that magnetic anisotropy depends on the shape, relative magnetic intensity, and proportion of the magnetic minerals.
\end{abstract}

\section{Introduction}

The magnetic anisotropy of rocks is sensitive to variations in both strain and composition. Total anisotropy of induced magnetic susceptibility (AMS) may be thought of as the average intrinsic anisotropy of each mineral phase, weighted by its susceptibility. Therefore, small variations of a highly susceptible phase can greatly skew the observed anisotropy [Henry, 1983]. Magnetic anisotropy is often interpreted as representing the preferred orientation of ferrimagnetic magnetite, but it has recently been shown that crystallographic orientation of paramagnetic silicates can significantly contribute to the anisotropy, especially in rocks with minimal magnetite content [Borradaile, 1987; Borradaile et al., 1987; Hrouda, 1987; Rochette, 1987].

Anisotropy of anhysteretic susceptibility (AAS), however, is a direct measure of the remanent magnetization fabric, recording only the ferro- and ferrimagnetic components [McCabe et al., 1985]. Because the signal from fine-grained magnetite is enhanced, anhysteretic susceptibility may yield a stronger, more reproducible signal than low-field susceptibility.

Henry [1983] and Henry and Daly [1983] described a graphical technique to identify compositional control of anisotropy and a mathematical technique to quantitatively separate the paramagnetic and ferrimagnetic fabrics. Their model is based on a two-component system, where total anisotropy increases with increased concentration of the

${ }^{1}$ Currently at University of Texas, Austin.

Copyright 1991 by the American Geophysical Union.

Paper number 91GL01418

0094-8534/91/91GL-01418\$3.00 more anisotropic mineral. There exists a linear relationship between the diagonal components $\left(\mathrm{k}_{\mathrm{ii}}\right)$ (the components in the $x, y$, and $z$ directions) of the AMS tensor $[K]$, and mean susceptibility ( $\left.k_{\text {mean }}\right)$ if composition rather than strain controls the variation in anisotropy. This relationship can be tested by least-squares regression analysis. The divergence of regression lines represents increasing anisotropy, and their intersection represents a hypothetical, isotropic component. Intersection at the origin is expected for a single magnetic phase; intersection off the origin signifies a twocomponent system. The geometry of the regression lines, therefore, constrains possible end-member phases.

Comparisons of $k_{i i}$ and $k_{\text {mean }}$ for a variety of lithologies have been interpreted as the mixing of a low susceptibility, weakly anisotropic matrix and a highly anisotropic ferrimagnet [Henry, 1983; Henry and Daly, 1983; Borradaile and Sarvas, 1990]. In such cases, anisotropy increases with mean susceptibility, in agreement with direct comparison of anisotropy and susceptibility [Rochette, 1987; Damm and Andrehs, 1988]. Likewise, Borradaile and Puumala [1989] have mixed oblate magnetite in a paramagnetic plasticene matrix, to show that anisotropy increases with magnetite concentration. On the other hand, Borradaile [1987] theoretically predicted that addition of detrital, rounded magnetite to anisotropic paramagnetic phyllosilicates would decrease the total anisotropy. The anisotropy, therefore, must depend on the relative shape, as well as concentration, susceptibility, and orientation of the components.

In this study, two paramagnetic minerals of differing shape and susceptibility were mixed with magnetite, then compacted at the same pressure to test the effect of composition on anisotropy. Samples were exposed to a magnetic field large enough to produce a lineation in the magnetite but not in the paramagnets. AMS and AAS were compared, to test if the two measurement techniques are able to differentiate the paramagnetic and ferrimagnetic fabrics.

\section{Methods}

Samples were prepared by combining the paramagnetic minerals, chlorite and $\mathrm{Mn}_{2} \mathrm{O}_{3}$, and the ferrimagnetic mineral magnetite. Natural chlorite (clinochlore, Wards Scientific, Rochester, NY) was chosen as the anisotropic paramagnet. Chlorite's easy plane of magnetization is its basal plane, which is also its cleavage plane. Samples were ground with a silicate mortar and pestle and agate ball-mill (to avoid metallic contamination), then sieved between 40 and 250 standard mesh $(0.058 \mathrm{~mm}$ to $0.42 \mathrm{~mm})$, large enough so that the sieved particles were visibly platy. Magnetite naturally intergrown with the chlorite was removed with a hand magnet; however, the samples retained a weak anhysteretic remanent magnetization $\left(2.5 \times 10^{-2}\right.$ 
$\mathrm{A} / \mathrm{m}$ ). Synthetic $\mathrm{Mn}_{2} \mathrm{O}_{3}$ (325 mesh $=44 \mathrm{~mm}$, Alfa Products) was used as a moderately susceptible, isotropic (presumably equant) paramagnet. It also had a weak anhysteretic remanence $\left(2.5 \times 10^{-2} \mathrm{~A} / \mathrm{m}\right)$, due to ferrimagnetic contamination. Magnetite was added as the ferrimagnet (30$40 \mu \mathrm{m}$, Wright Industries Brooklyn, NY). Magnetite's anisotropy is strongly shape-controlled, and scanning electron microscopy revealed a distribution of grain shapes from equant to platy in this magnetite sample (Madeline $J$. Bryant, personal communication). $\mathrm{CaF}_{2}$ was added as an inert filler to samples with $\mathrm{Mn}_{2} \mathrm{O}_{3}$.

The desired proportions of each component were manually mixed to form each sample. The mixture was loosely deposited into a brass collar, then exposed to a $0.1 \mathrm{~T}$ field inside an electromagnet for one minute to align the magnetite. A hydraulic press compacted the sample into an 8 $\mathrm{cm}^{3}$ cube at $27.56 \mathrm{MPa}$. The field and pressing directions were recorded on the plastic boxes housing the samples. All samples were oriented such that the field direction was at $0^{\circ}$ azimuth and horizontal, and the pressing direction was vertical, so that the imposed magnetic lineation was in the plane of foliation produced by compaction.

AMS was measured in a susceptibility bridge with an applied magnetic field of approximately $1 \mathrm{mT}$. A microcomputer-controlled robotic arm rotated the sample so that the susceptibility was measured at $1.8^{\circ}$ intervals about three orthogonal sample axes. Computer programs based on calculations adapted from Girdler [1961] calculated the bestfit AMS tensor for each sample. Susceptibilities of these samples ranged from $10^{-4}$ to $10^{-2} \mathrm{SI}$, at least two orders of magnitude greater than the sensitivity of the instrument $\left(10^{-6}\right.$ SI). Multiple measurements of the samples yielded highly reproducible data, so that the standard deviation was approximately $1 \%$ of the susceptibility measurement.

AAS was measured according to the technique of McCabe et al. [1985]. Samples were first demagnetized in a $100 \mathrm{mT}$ field, then exposed to a peak alternating field of 80 $\mathrm{mT}$ with a coaxial direct field of $0.1 \mathrm{mT}$. The remanent magnetization was subsequently measured in a cryogenic magnetometer. This procedure was repeated along nine directions corresponding to $45^{\circ}$ intervals about three orthogonal axes. Tensors were fit to these data using least squares.
We evaluated the quality of the fit of the tensors by comparing the ratio of the root-mean-squares of the residuals (observed values minus calculated values) to the root-meansquares of the susceptibility measurements [McCabe et al., 1985; Jackson et al., 1989]. This ratio, less than $1.5 \%$ for all samples, in turn was compared with the percent anisotropy $\left\{100\left(k_{\max }-k_{\min }\right) / k_{\text {mean }}\right\}$. The tensors were well resolved. The ratio \{(residuals/ susceptibility)/ \%anisotropy\} was generally less than 0.1 . The ratio $\{$ (residuals/ susceptibility)/\%anisotropy $\} \approx 0.2$ for samples with low anisotropy $\left(\mathrm{Mn}_{2} \mathrm{O}_{3}+0 \%\right.$ magnetite and all the samples mixing only chlorite and $\mathrm{Mn}_{2} \mathrm{O}_{3}$ ).

\section{$\mathrm{Mn}_{2} \mathrm{O}_{3}$ and Magnetite}

Samples of $0.0,0.25,0.5$, and 1.0 weight percent magnetite in $\mathrm{Mn}_{2} \mathrm{O}_{3}$ were combined to test the effect of adding an anisotropic ferrimagnet to a relatively isotropic paramagnet. Minimum and maximum orientations deviate from the pressing and field directions for the sample with no added magnetite, indicating that the ferrimagnetic contaminant in the $\mathrm{Mn}_{2} \mathrm{O}_{3}$ is isotropic, unrotated by the deformation (Figure 1a). Minima for both AMS and AAS are steep, in agreement with the pressing direction, and define a pole to the subhorizontal foliation. The maxima, representing the magnetic lineation, lie in the field direction. There are no systematic differences between AMS and AAS orientations, nor are there systematic changes in orientation with addition of magnetite.

The shape and relative magnitude of AMS and AAS ellipsoids are illustrated on the Flinn diagram (Figure 1b). Susceptibility anisotropy is negligible for the sample without added magnetite. Remanent anisotropy of this sample is higher, without a pronounced lineation or foliation. All the AMS measurements fall within the prolate field. Addition of magnetite increases the magnitude of the anisotropy; however, there is no systematic change in the shape of the AMS ellipsoids with increasing magnetite content. Remanent magnetization is more anisotropic than induced magnetization for all samples. There are no systematic changes in AAS ellipsoid shape or magnitude with addition of magnetite. Moreover, there are no systematic differences between AMS and AAS for any given sample. a)

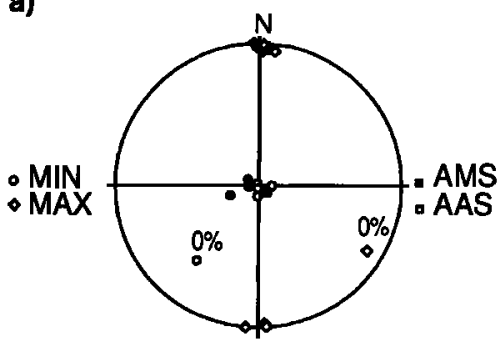

b)

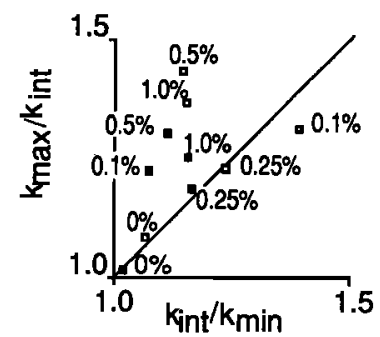

\section{(1)}

Mn ${ }_{2} Q_{3}$ AND MAGNETITE 
Directional components of AMS $\left(\mathrm{k}_{\mathrm{ii}}\right)$ plotted against mean susceptibility $\left(\mathrm{k}_{\text {mean }}\right)$ yield regression lines with high correlation coefficients $\left(r^{2}>0.99\right)$ (Figure $\left.1 c\right)$. Error of each term (RMS residuals/RMS susceptibility) was less than $1.5 \%$, such that error bars are smaller than the symbols plotted. Regression lines intersect at the point representing the sample with no added magnetite, verifying its isotropic fabric. Regression lines diverge with increasing susceptibility, indicating that the susceptibility anisotropy increases with addition of magnetite.

\section{Chlorite and Magnetite}

Samples with $0.0,0.1,0.25,0.5$, and 1.0 weight percent magnetite in chlorite were prepared to test the effect of adding a ferrimagnet to a platy paramagnet. Two samples of each composition were prepared (only one sample with $0.1 \% \mathrm{Fe}_{3} \mathrm{O}_{4}$ ) to illustrate heterogeneity of the parent materials and experimental variability.

Magnetic minima are in the pressing direction and maxima are in the field direction for both AMS and AAS (Figure 2a). There are no systematic changes in orientation with composition.

Chlorite without added magnetite has a weak AMS foliation (Figure 2b). At low magnetite concentration (0.1\%) the AMS ellipsoids plot in the oblate field and trend slightly towards the prolate field, then become more anisotropic with addition of magnetite. Remanent magnetization is uniformly more anisotropic than induced magnetization, and AAS ellipsoids mimic the AMS trend towards the prolate field with small additions of magnetite. Note that without added magnetite, AAS records a strong foliation, due to magnetite contamination in the original chlorite. To correct for the contaminant's magnetic fabric, the AAS tensor for this sample was subtracted from other AAS tensors in the trial (Figure 2c). The resultant tensors are similar to the original ones, because the magnitude of the contaminant's remanent magnetization is weak, even though it is relatively anisotropic.

A strong linear relationship exists between AMS directional components and mean susceptibility (Figure 2d). Regression coefficients $\left(\mathrm{r}^{2}\right)$ are greater than 0.99 and error of each term is less than $1.0 \%$. Regression lines intersect off the origin, as predicted for a two component system [Henry 1983]. Anisotropy increases with addition of magnetite. Susceptibility differences between samples of the same weight percent magnetite illustrate the heterogeneity of the natural chlorite.

\section{Chlorite and $\mathrm{Mn}_{2} \mathrm{O}_{3}$}

Samples with $5,10,33$, and 50 weight percent $\mathrm{Mn}_{2} \mathrm{O}_{3}$ in chlorite were prepared to test the effect of an isotropic, moderate susceptibility paramagnet with a platy, low susceptibility paramagnet. Since no ferrimagnet was added, these samples were not exposed to a field, and only AMS was measured.

The orientations of principal susceptibility components change with composition. The minima generally become shallower and the maxima steepen with increasing content of $\mathrm{Mn}_{2} \mathrm{O}_{3}$ (Figure 3a). AMS ellipsoids become less anisotropic and less oblate with addition of $\mathrm{Mn}_{2} \mathrm{O}_{3}$ (Figure 3b).

Directional components of susceptibility show a strong linear correlation with mean susceptibility (Figure 3c). Regression coefficients $\left(\mathrm{r}^{2}\right)$ are greater than 0.99 , and the error of each term is less than $1.0 \%$. The $\mathrm{k}_{\mathrm{xx}}$ and $\mathrm{k}_{\mathrm{yy}}$ regression lines are statistically coincident, due to the oblate (compaction) fabric. The $95 \%$ confidence limit for the slope of the $\mathrm{k}_{\mathrm{zz}}$ regression line lies outside of the $95 \%$ confidence limit of the other two lines, demonstrating that it is statistically distinct. Anisotropy decreases with increasing susceptibility, controlled by the addition of isotropic $\mathrm{Mn}_{2} \mathrm{O}_{3}$.

\section{Discussion and Conclusions}

Although the experiments were designed to produce separate ferrimagnetic and paramagnetic fabrics, magnetite evidently dominated the susceptibility signal even at low concentrations: AMS and AAS were coaxial, the fabrics were predominantly prolate, remanent magnetization was consistently more anisotropic than induced magnetization, and the shape of AAS ellipsoid does not differ systematically from the AMS ellipsoid with addition of magnetite. Relative oblateness of fabrics in the trials with chlorite, as opposed to

\section{CHLORITE AND MAGNETITE}
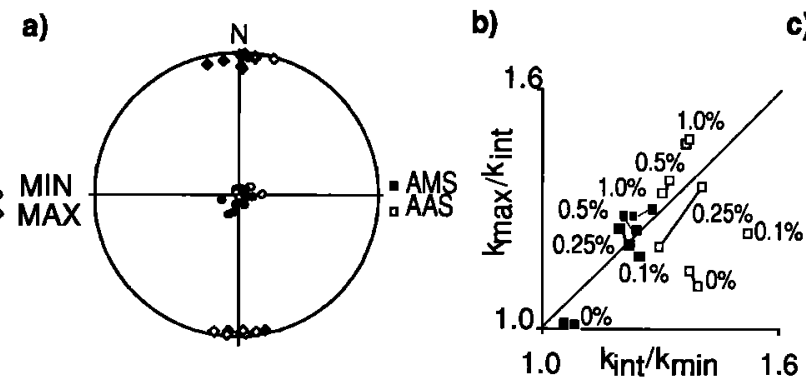

c)

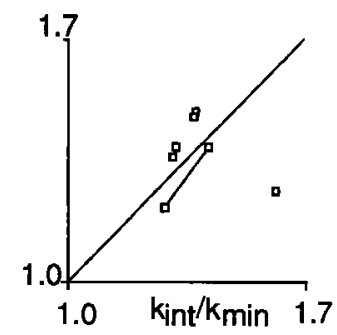

d) 0

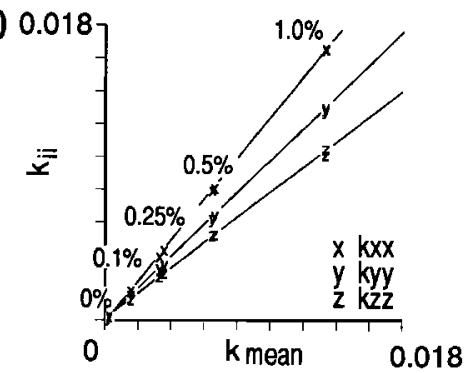

Fig. 2. Weight percent magnetite indicated. a) Stereographic projections, average of samples with like composition. b) Flinn diagram. Samples of like composition are connected. c) Flinn diagram corrected for ferrimagnetic contamination of the chlorite. d) Least-squares regression lines correlating directional susceptibility $\left(\mathrm{k}_{\mathrm{ii}}\right)$ and mean susceptibility $\left(\mathrm{k}_{\text {mean }}\right)$. 

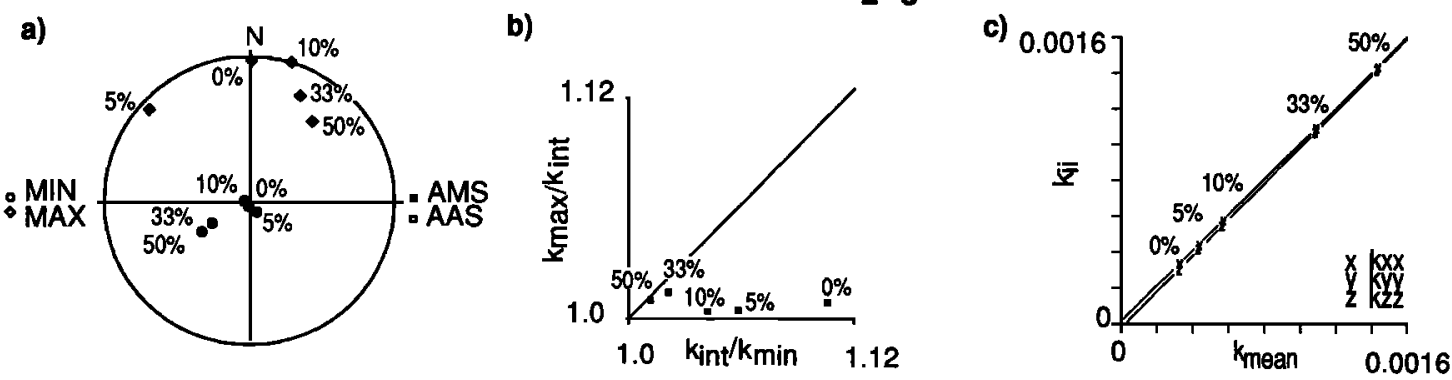

Fig. 3. Weight percent $\mathrm{Mn}_{2} \mathrm{O}_{3}$ indicated. a) Stereographic projections. b) Flinn diagram. c) Leastsquares regression lines correlating directional susceptibility $\left(\mathrm{k}_{\mathrm{ii}}\right)$ and mean susceptibility $\left(\mathrm{k}_{\mathrm{m}} \mathrm{man}\right)$.

the predominantly prolate fabric of trials with $\mathrm{Mn}_{2} \mathrm{O}_{3}$, is evidence for the (weak) paramagnetic contribution to the anisotropy. Further trials with even lower concentrations of magnetite might yield more distinct paramagnetic and ferrimagnetic fabrics.

These experiments demonstrate that magnetic anisotropy depends on the shape and proportion of the more highly susceptible mineral, verifying Henry's mixing model. The intersection of the regression lines represents the susceptibility of the isotropic mineral, and the geometry of the plot critically depends on the shape of the high susceptibility mineral. Thus, the anisotropy increases with increasing susceptibility if the high susceptibility component is anisotropic magnetite, and decreases if it is isotropic $\mathrm{Mn}_{2} \mathrm{O}_{3}$. Additionally, the anisotropy can be controlled by the relative proportions of two paramagnets, important for lithologies lacking a ferrimagnet.

The general results have important implications for natural samples. Compositional control of anisotropy may be recognized by $k_{i i}$ versus $k_{\text {mean }}$ plots, and the geometry of the regression lines may be used to help model the magnetic components. Relative shapes and magnitudes of AMS and AAS ellipsoids illustrate qualitative differences between the bulk susceptibility and ferrimagnetic remanent fabric, which are likely related to the shape of the magnetic minerals. These procedures do not produce unique models for magnetic mineralogy, but combined with independent tests of the mineralogy (both magnetic and petrologic), they do constrain interpretation of the magnetic fabric.

Acknowledgements. This work was supported by NSF EAR 88-04-820. We thank Jim Marvin for helping to design the experiments, and Peter Hudleston and Subir Banerjee for their advice throughout the study.

\section{References}

Borradaile, G.J., Anisotropy of magnetic susceptibility: Rock composition versus strain, Tectonophysics. 156, 1-20, 1987.

Borradaile, G.J. and C. Alford, Relationship between magnetic susceptibility and strain in laboratory experiments, Tectonophysics, 133, 121-135, 1987.

Borradaile, GJ. and C. Alford, Experimental shear zones and magnetic fabrics, J. Struct. Geo.. 10, 895-904, 1988.
Borradaile, G.J. and M.A. Puumala, Synthetic magnetic fabrics in a plasticene medium, Tectonophysics, $164.73-78$, 1989.

Borradaile, G.J. and P. Sarvas, Magnetic susceptibility fabrics in slates: structural, mineralogical and lithological influences, Tectonophysics, 172, 21-222, 1990.

Borradaile, G.J., W. Keeler, C. Alford, and P. Sarvas, Anisotropy of magnetic susceptibility of some metamorphic minerals, Physics Earth Planet. Int. 48, 161-166, 1987.

Damm, V. and G. Andrehs, Variations of petromagnetic fabric parameters of some Antarctic metamorphic rocks in the light of changes in the mineralogical and geochemical content, Physics Earth Planet Int., 51, 313-319, 1988.

Girdler, R.W., The measurement and computation of anisotropy of magnetic susceptibility in rocks, Geo. $\underline{\text { J. Royal }}$

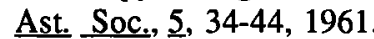

Henry, B., Interpretation quantitative de l'anisotropie de susceptibilite magnetique, Tectonophysics, 91, 165-177, 1983.

Henry, B. and L. Daly, From qualitative to quantitative magnetic anisotropy analysis: the prospect of finite strain calibration, Tectonophysics, $28,327-336,1983$.

Hrouda, F., Mathematical model relationships between the paramagnetic anisotropy and strain in rocks, Tectonophysics, 142, 323-327, 1987.

Jackson, M., D. Sprowl, and B. Ellwood, Anisotropies of Partial Anhysteretic Remanence and Susceptibility in compacted Black Shales, Geo. Res. Let. 16, 1063-1066, 1989.

McCabe, C., M. Jackson,, and B. Ellwood, Magnetic anisotropy of the Trenton Limestone, Geo. Res. Let., 12, 333-336, 1985.

Rochette, P., Magnetic susceptibility of the rock matrix related to magnetic fabric studies, J. Struct. Geo., 2. 1015$1020,1987$.

M. Jackson, Dept. Geology and Geophysics, University of Minnesota, Minneapolis, MN, 55455.

M. Johns, Department of Geological Sciences (51640), University of Texas, Austin, Texas, 78713.

(Received: January 21, 1991:

Accepted: April 25, 1991.) 